\title{
Der Graben zwischen Naturheillkunde und Schulmedizin schließt sich langsam, aber nicht nur, weil die Medizin offener geworden wäre
}

\author{
Patienten können sich immer besser über Therapien informieren - \\ Dies wird das Interesse an seriöser Naturheilkunde weiter steigen lassen \\ Ein Gespräch mit Prof. Gustav Dobos
}

Sie haben sich schon früh mit komplementären Behandlungsverfahren beschäftigt, was hat Ihr Interesse geweckt?

Als Nephrologe, also Nierenspezialist, habe ich viele Patienten auf der Intensivstation oder in der Dialyse gesehen, die wegen chronischer Schmerzen viele Medikamente nahmen und dadurch ihre Nieren zerstört haben. Ich dachte mir: Es muss doch möglich sein, diese Menschen VORHER so zu therapieren, dass die Nieren nicht geschädigt werden.

Während des Medizinstudiums haben Sie auch in der VR China Akupunktur studiert. Wie hat sich Ihre Sicht auf die Naturheilkunde in Deutschland nach dem ChinaAufenthalt verändert?

Ich habe in China gesehen, dass das westliche Medizinverständnis nicht das einzig funktionierende ist - sondern dass die Chinesen in ihrer traditionellen Heilkunde erstaunliches diagnostisches und therapeutisches Wissen vereinen. In China übrigens sind westliche und traditionelle Medizin keine Gegensätze, sondern werden häufig miteinander kombiniert.

Sie leiten seit 1999 die Klinik für Naturheilkunde und Integrative Medizin in Essen. Welchen Stellenwert hatten Naturheilkunde und Integrative Medizin als Sie damals begannen?
Wir mussten uns gegen große Widerstände behaupten und wurden als Modellklinik des Landes NRW auch besonders streng evaluiert. Damals schickte man uns Patienten, die die anderen Ärzte für menschlich schwierig und schwer behandelbar hielten, um uns zu ärgern.

Was hat sich seitdem verändert?

Heute schicken uns Kollegen Patienten, bei denen sie mit ihren Therapien nicht weiterkommen, um von unserem Ansatz zu lernen. Und viele schicken auch ihre eigenen Angehörigen oder kommen selbst als Patient, wenn notwendig.

Gibt es für Sie Verfahren der Naturheilkunde, die sich als besonders wichtig herausgestellt haben?

Wir therapieren chronische Erkrankungen das betrifft bundesweit mehr als die Hälfte aller stationär oder ambulant behandelten Patienten. Bei diesen Krankheiten - z.B. Bluthochdruck, Rheuma, chronische Darmerkrankungen oder Allergien - setzt die Schulmedizin Medikamente ein, die kurzfristig Symptome lindern und mitunter - etwa bei einem Asthmaanfall - auch lebensrettend sein können. Langfristig eingenommen aber können sie selbst medizinische Probleme hervorrufen, belasten die Nieren, schädigen die Leber oder verstärken das Krebsrisiko. Bei Naturheilkunde und

\section{Zusammenfassung}

Prof. Gustav Dobos hat den Stiftungslehrstuhl für Naturheilkunde an der Universität Duisburg-Essen inne und leitet die Klinik für Naturheilkunde und Integrative Medizin, Kliniken Essen-Mitte. Der Internist, Nephrologe und Intensivmediziner mit den Zusatzbezeichnungen Akupunktur, Naturheilkunde, Physikalische Therapie und Chirotherapie ist Verfechter einer Integrativen Medizin, die die Denkweisen der konventionellen Medizin und der Naturheilkunde in sinnvoller Weise verbindet.

Warum Offenheit, Respekt und Neugier für die Integrative Medizin genauso wichtig sind wie professionelles Wissen und die Bedeutung einer seriösen Naturheilkunde in Zukunft weiter zunehmen wird, erzählt Dobos im zkm-Interview.

Komplementärmedizin geht es v.a. um den langfristigen Verlauf: So viel Medikamente wie nötig, so wenig wie möglich. Unser Ziel ist es, die Selbstheilungsprozesse im Körper zu wecken - das kann durch Heilfasten, Phytotherapie, Akupunktur oder auch Meditation geschehen. Am besten wissenschaftlich untersucht sind Akupunktur und die sog. Mind-Body-Medizin - mentale Techniken zur Entspannung, Meditation, Yoga und Achtsamkeitspraktiken. 


\section{In der Integrativen Medizin geht} es um die Verbindung von Schulmedizin und Naturheilkunde - die Integration beider Denkweisen.

Rein konzeptionell habe ich zu Beginn systematisch die wissenschaftliche Literatur zum Thema Komplementärmedizin analysiert und habe die besten komplementärmedizinischen Zentren der Welt, wie beispielsweise die Scripps Clinic in San Diego, die Harvard Medical School und das Memorial Sloan Kettering Hospital in New York City persönlich aufgesucht und mit den Leitern unterschiedliche Strategien diskutiert. Ziel war es von Anfang an, ein Zentrum für wissenschaftlich geprüfte Naturheilkunde aufzubauen.

Prof. Gustav Dobos

Wo sehen Sie Entwicklungschancen insbesondere für Kliniken mit NHV und CAM? Die Integrative Medizin, wie sie seit 20 Jahren in den USA praktiziert wird, ist der Weg der Zukunft. Die Verbindung von moderner Biomedizin mit traditionellen Heilverfahren auf Evidenzbasis, also wissenschaftlich überprüft. In den USA haben z. B. alle großen Krebskliniken - wie das Sloan Kettering Cancer Center in New York oder die Harvard Medical School in Boston - Abteilungen für Integrative Onkologie. Vom Tag der Diagnosestellung an werden Patienten auch komplementärmedizinisch begleitet. Das ist bei Krebs besonders wichtig, da nach Schätzungen an die $70 \%$ der Krebspatienten sich ohnehin „nebenbei“ selbst therapieren, mit Kräuterauszügen oder Vitamincocktails. Die wenigsten sagen das ihrem Arzt, das ist ein erhebliches Risiko. Denn so etwas scheinbar Harmloses wie Johanniskraut gegen leichte bis mittelschwere Depressionen kann eine Chemotherapie in ihrer Wirksamkeit abschwächen. Wir machen in Essen sehr gute Erfahrungen mit mehreren Pilotprojekten im Bereich Gynäkologischer Integrativer Onkologie (Brust-, Gebärmutterhals- und Eierstockkrebs). Es wird Zeit, dass die Onkologie die zum Teil erheblichen Nebenwir- kungen von Krebsbehandlungen ernst nimmt und etwas dagegen tut.

Sie sind seit 2004 Inhaber des Lehrstuhls für Naturheilkunde an der Uni DuisburgEssen. Was hat Sie an dieser Position besonders gereizt?

Mich hat besonders die Forschung gereizt nachweisen zu können, dass Naturheilkunde und Traditionelle Heilverfahren helfen. Außerdem konnten wir mit unserem Team ein in Deutschland innovatives integriertes Behandlungsmodell entwickeln, das multiprofessionell und sektorenübergreifend ist. Und natürlich ist es wichtig, die Ergebnisse an die junge Medizinergeneration weiterzugeben.

\section{Wie haben Sie Ihre Strategie entwickelt?} Ausgangspunkt ist die Salutogenese, die Gesundheitsforschung, die von Aaron Antonovsky Mitte des 20. Jahrhunderts begründet wurde. Nicht nur die Krankheit selbst, sondern auch die Ressourcen für Gesundheit, die der Patient oder die Patientin mitbringen, werden beachtet und in Frage gestellt. Diese versuchen wir zu stärken. Das Wichtigste am Heilungsprozess macht der Patient selbst und der Arzt unterstützt den Prozess mit naturheilkundlichen Therapien.
Welches sind die Schwerpunkte des Lehrstuhls?

Die aktuellen Schwerpunkte des Lehrstuhl sind die „Integrative Onkologie“, d.h. die kombinierte Behandlung von Krebspatienten, die Mind-Body-Medizin sowie die Entwicklung von wissenschaftlichen Leitlinien durch systematische Analyse der wissenschaftlichen Fachliteratur in Zusammenarbeit mit den jeweiligen Fachgesellschaften.

Durch die wissenschaftlichen Erkenntnisse können wir vielen Menschen helfen und dazu beitragen, dass die Naturheilkunde endlich in die Regelversorgung integriert wird, zum Wohle des Patienten.

Ein wissenschaftlich neuer Bereich ist die Konzeptentwicklung und Umsetzung von komplementärmedizinischen Therapieansätzen in der Kinder- und Jugendmedizin. Dabei entwickelten wir gemeinsam mit der Universitätsklinik, dem Hauner'schen Kinderspital in München ein ambulantes und stationäres Konzept zur Behandlung von Kindern. Unterstützt wird das Projekt von der Karl und Veronica Carstens-Stiftung.

Gibt es Forschungsbereiche, die Sie besonders spannend finden oder die Sie überrascht haben? 
Die Integrative Onkologie ist sowohl vom klinischen wie auch vom wissenschaftlichen Standpunkt her ein sehr spannendes Feld, weil den Patienten damit sehr gut geholfen werden kann, mit der Erkrankung fertig zu werden. Die dazu aktuell durchgeführten Studien sind noch nicht abgeschlossen.

Wir haben aber eine Vielzahl von Studien zur Behandlung von chronischen Schmerzen durchgeführt. So kamen unter anderem Blutegel, Akupunktur, Kohlblätter, Yoga, Meditation, Gua Sha und Schröpfen zum Einsatz. Das Überaschende dabei war, dass naturheilkundliche Verfahren teilweise wirksamer waren als konventionelle, bei weniger Nebenwirkungen.

Wo sehen Sie Schwierigkeiten bei der komplementärmedizinischen Forschung?

Generell ist in der Forschung zu Komplementärmedizin schwierig, das Vorgehen in klassischen Doppelblindverfahren zu testen, weil die Beziehungsebene zwischen Behandler und Behandeltem eine große Rolle spielt und sich kaum standardisieren lässt. Grundsätzlich schwierig in der komplementärmedizinischen Forschung ist die Finanzierung. Offizielle staatliche Förderung z.B. durch die Deutsche Fördergemeinschaft ist - im Gegensatz zu den USA, wo von den National Institutes of Health (NIH), der obersten Gesundheitsbehörde, jährlich für 120 Mio. Dollar Projekte gefördert werden nicht erhältlich.

Wie sehen Sie das Verhältnis von Ärzten zu anderen Berufen, die CAM anwenden, insbesondere zu Heilpraktikern? Wie sollte die Zusammenarbeit sein und wo sind die Grenzen?

Das Feld ist komplex: Es gibt vereinzelt gut qualifizierte Heilpraktiker, aber weniger als ein Viertel derjenigen, die die Heilpraktikerprüfung ablegen, praktizieren regelmäßig und haben ausreichende Erfahrung. Wichtig wäre, dass Patienten mit ihren Ärzten offen über alles reden können - auch über das, was der Heilpraktiker ihnen vorschlägt. Häufig fehlt es bei den Medizinern an Zeit und Bereitschaft. Und Hintergrundwissen darüber, welche Wirkungen z. B. Heilkräuter und andere naturheilkundliche Therapien haben. Für Onkologen haben wir deshalb ein Fort/Weiterbildungs-Curriculum entwickelt. Es ist notwendig, dass Ärzte sich hier weiterbilden - die Patienten machen sonst, was sie glauben oder was man ihnen einzu-

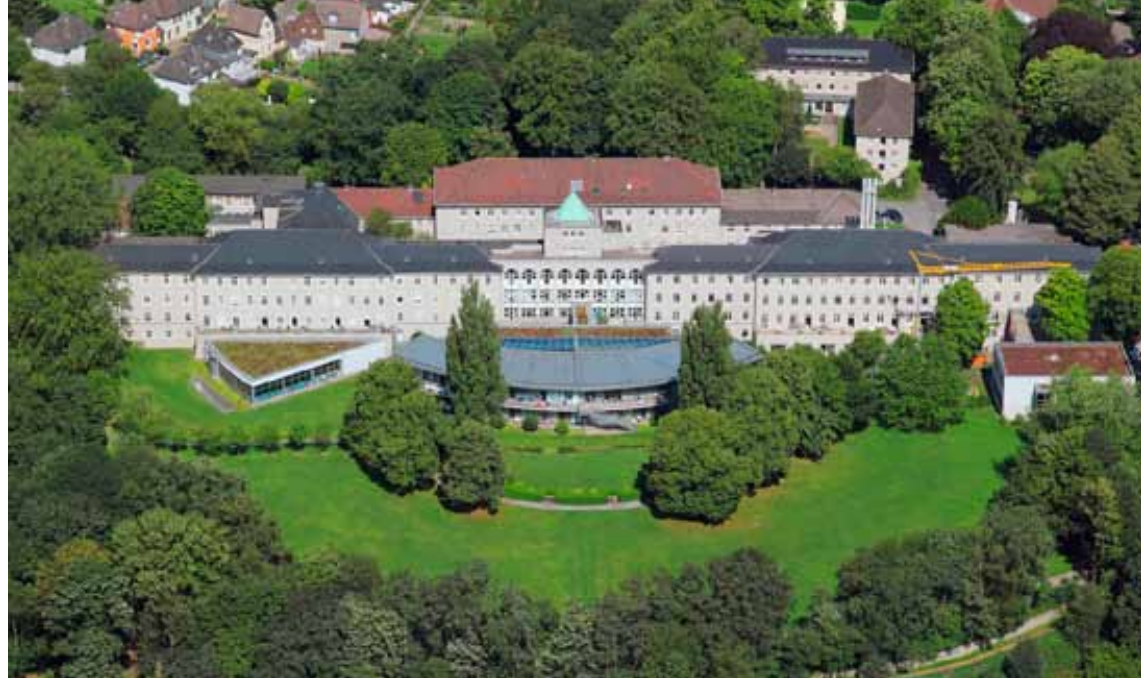

Abb. 1 Die Klinik für Naturheilkunde und Integrative Medizin wurde 1999 als Modelleinrichtung ins Leben gerufen. Sie bietet ein breites Spektrum naturheilkundlicher Behandlungen mit den diagnostischen und therapeutischen Möglichkeiten eines modernen Krankenhauses.

reden versucht. Und skrupellose Heilsversprecher gibt es genügend auf dem Markt.

\section{Naturheilkunde - Komplementärmedizin -} Integrative Medizin: Gibt es eine ideale Bezeichnung?

Es geht um die Verbindung, also die Integration beider Denkweisen, deshalb Integrative Medizin.

\section{Was integriert eine Integrative Medizin?} Ist das lehrbar?

Selbstverständlich. Wir bilden Ärzte und Pflegende aus und letztlich schulen wir auch die Patienten selbst, wie sie mit ihrer Krankheit am besten umgehen können. Eine wichtige Botschaft ist dabei, nicht nur das Leiden, sondern die Patientin oder den Patienten im Blick zu haben, ihre oder seine individuellen Fragestellungen und Ressourcen. Das ist der größte Schritt, den z. B. junge Mediziner machen müssen, wenn sie zu uns kommen - denn sie sind geschult worden, Symptome und Krankheiten zu therapieren, weniger den Menschen, zu dem sie gehören. Dazu gehören Offenheit, Respekt und Neugier für das, was unsere Patienten mitbringen. Dazu gehört aber auch das professionelle Wissen, welches therapeutische Vorgehen konventionell, als auch naturheilkundlich bei genau diesem Patienten den meisten Erfolg verspricht. Integrative Medizin bedeutet in dem Sinne auch Zweisprachigkeit und das ist auch die Schwierigkeit beim Erlernen. Das kann z. B. bei einem Prostatakrebs-Patienten eine Bestrahlung sein, bei einem anderen Abwarten und Lebensstiländerungen. Integrieren bedeutet hier, alle Optionen gemeinsam zu prüfen und sorgsam abzuwägen. Im Idealfall bedeutet ,Integrative Medizin“ die Kombination von guter Schulmedizin mit wissenschaftlicher Naturheilkunde.
Hat die Naturheilkunde inzwischen den Stellenwert, der ihr zusteht? Schließt sich der Graben zwischen Naturheilkunde und Schulmedizin?

Der Graben schließt sich langsam, aber nicht, weil die Medizin offener geworden wäre. Es gibt dort immer noch sehr viel Neid auf die Naturheilkunde, weil die Zustimmung vonseiten der Patientinnen und Patienten groß ist und weiter wächst. Gleichzeitig wünscht sich die Bevölkerung, das zeigen alle Umfragen, die Verbindung von Schulmedizin und traditionellen Heilverfahren.

Die demografische Entwicklung wird die Zahl der chronischen Krankheiten wie auch Krebs enorm steigen lassen. Die individualisierte Biomolekularmedizin, die zum Teil Erstaunliches vollbringt, steckt trotzdem in der Krise: Sie wird sich nicht in breitem Maßstab finanzieren lassen. Die Pharmaindustrie zieht sich aus allen Bereichen, wo keine großartigen Gewinne zu erwarten sind, zurück. Die Zahl der Ärzte geht drastisch zurück und der Informationsstand der Patienten wird aufgrund neuer Medien immer besser. Die Medizin ist in weiten Bereichen keine geheime Wissenschaft mehr und Patienten können sich immer besser darüber informieren, welche Therapien für sie geeignet sind. Dies wird meiner Erfahrung nach das Interesse an seriöser und wissenschaftlich geprüfter Naturheilkunde in $\mathrm{Zu}$ kunft noch weiter ansteigen lassen.

\section{Herzlichen Dank für das Interview.}

Interview: Anke Niklas

Online zu finden unter

http://dx.doi.org/10.1055/s-0034-1390678 